\title{
TET-catalyzed 5-methylcytosine hydroxylation is dynamically regulated by metabolites
}

\author{
Cell Research (2014) 24:1017-1020. doi:10.1038/cr.2014.81; published online 27 June 2014
}

\section{Dear Editor,}

DNA methylation suppresses gene expression and plays a broad and important role in diverse biological processes such as development and tumor suppression [1, 2]. DNA methylation is generally thought to be relatively stable and heritable. This view is recently changed by the discovery of TET (ten-eleven translocation) family of dioxygenases which catalyze three sequential oxidation reactions: converting $5 \mathrm{mC}$ first to $5 \mathrm{hmC}$, and then 5 -formylcytosine (5fC), and finally 5-carboxylcytosine (5caC) [3-7]. A subsequent decarboxylation of $5 \mathrm{caC}$ could then lead to DNA demethylation [3]. While the biological function and catalytic mechanism of TET enzymes are being extensively investigated, much less is currently known about their regulation. TET proteins are the members of $\mathrm{Fe}(\mathrm{II}) / \alpha$-ketoglutarate $(\alpha-\mathrm{KG})$-dependent dioxygenases, which require $\mathrm{Fe}(\mathrm{II})$ as a metal cofactor and $\alpha-K G$ as a co-substrate $[8,9]$. In various types of tumors, pathological accumulation of three metabolites, 2-HG, succinate and fumurate, that share structural similarity with $\alpha-K G$, results in a competitive inhibition of $\alpha-K G-$ dependent TET activity, leading to a hypermethylation phenotype $[10,11] . \alpha-K G$ is produced and consumed mainly by four different metabolic pathways: as a key intermediate in the TCA cycle for energy metabolism, as an entry point for several 5-carbon amino acids to enter the TCA cycle (anaplerosis) after being converted into glutamate, being reduced back to isocitrate and then citrate for the eventual synthesis of acetyl-CoA, and being used as a co-substrate for multiple $\alpha-K G$-dependent dioxygenases. These features of $\alpha-K G$ led us to investigate how a change in energy metabolism may influence TET activity in vivo.

We administered glucose in mice via an intraperitoneal injection protocol, which resulted in a rapid increase in blood glucose levels, reaching peak values at $t=30 \mathrm{~min}$ after injection, followed by a recovery at $t=60 \mathrm{~min}$ post injection (Figure 1A and Supplementary information, Figure S1A). GC-MS analysis revealed a rapid increase in hepatic $\alpha-K G$ levels that paralleled with the dynamic pattern of blood glucose, reaching peak values at $t=30$ min and returning to normal level at $t=60 \mathrm{~min}$ post injection (Figure 1B, Supplementary information, Figure S1B). Strikingly, genome-wide $5 \mathrm{hmC}$ levels were rapidly increased in various mouse tissues (the liver, kidney, and muscle) within 30 min of glucose injection as determined by dot-blot analysis (Supplementary information, Figures S1C). LC-MS/MS analysis confirmed that global $5 \mathrm{hmC}$ level was rapidly increased by 1.4 -fold $(P<0.01)$ in mouse livers within 30 min after glucose injection (Figure 1C and Supplementary information, Figures S1D). Notably, global $5 \mathrm{hmC}$ level rapidly declined/recovered at $t=60$ min post glucose injection (Figure 1C), which may at least in part be due to the decrease of $\alpha-K G$ after glucose withdrawl. Global $5 \mathrm{mC}$ levels were not changed by glucose injection (Supplementary information, Figure $\mathrm{S} 1 \mathrm{E})$. Global $5 \mathrm{fC}$ levels in the mouse liver were elevated slightly at $t=30 \mathrm{~min}$ after glucose injection, but unlike those of $5 \mathrm{hmC}$, continuously increased and reached a near-significant increase by $26.7 \%$ at $t=60 \mathrm{~min}$ after glucose injection (Supplementary information, Figure $\mathrm{S} 1 \mathrm{E}) .5 \mathrm{caC}$ levels were below the threshold of reliable detection (data not shown).

Moreover, we found that high levels of blood glucose and hepatic $\alpha-K G$ were maintained for an additional 30 $\min (t=30+30 \mathrm{~min})$ after repeated injection (Figure 1A, $1 \mathrm{~B}$ and Supplementary information, Figure S1A). Global $5 \mathrm{hmC}$ level was maintained at a high level in various mouse tissues at $t=30+30 \mathrm{~min}$ after repeated glucose injections as determined by both dot-blot and LC-MS/MS analyses (Figures 1C and Supplementary information, Figure S1C). Global 5mC levels were not substantially changed (Supplementary information, Figure S1E), while 5fC levels were significantly $(P<0.05)$ increased at $t=$ $30+30 \mathrm{~min}$ after repeated glucose injections as compared to $t=0 \mathrm{~min}$ (Supplementary information, Figure S1E). The mRNA and protein levels of Tet 2 and Tet3 were not altered in mouse livers after glucose shock (Supplementary information, Figure S1F). Moreover, Tet3 protein was predominantly in the nuclei of mouse hepatocytes at $t=60 \mathrm{~min}$ after single or repeated glucose injection, in- 
A

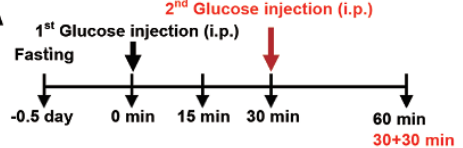

D

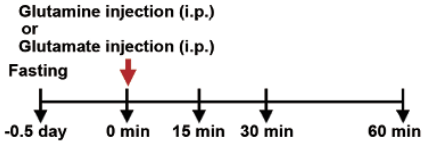

B
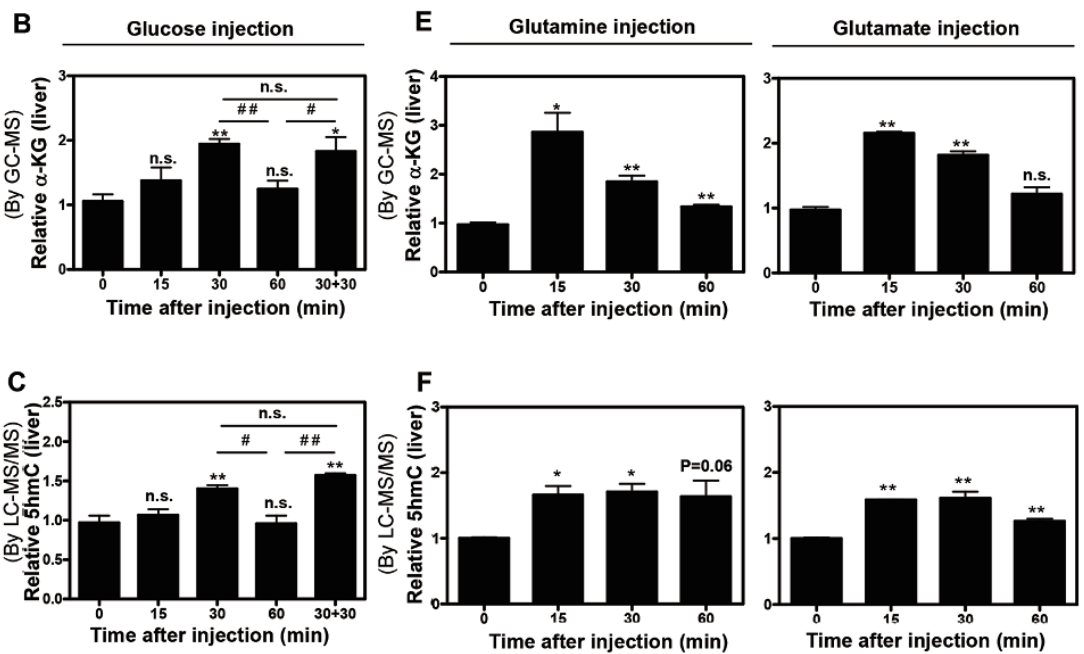

$\mathbf{J}$

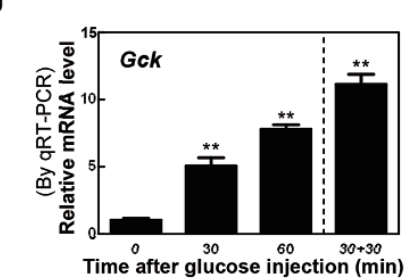

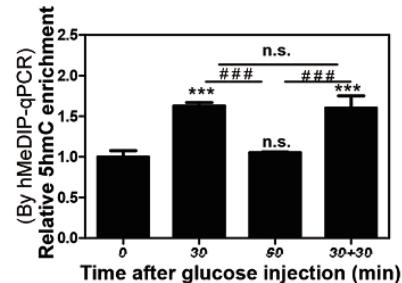

G

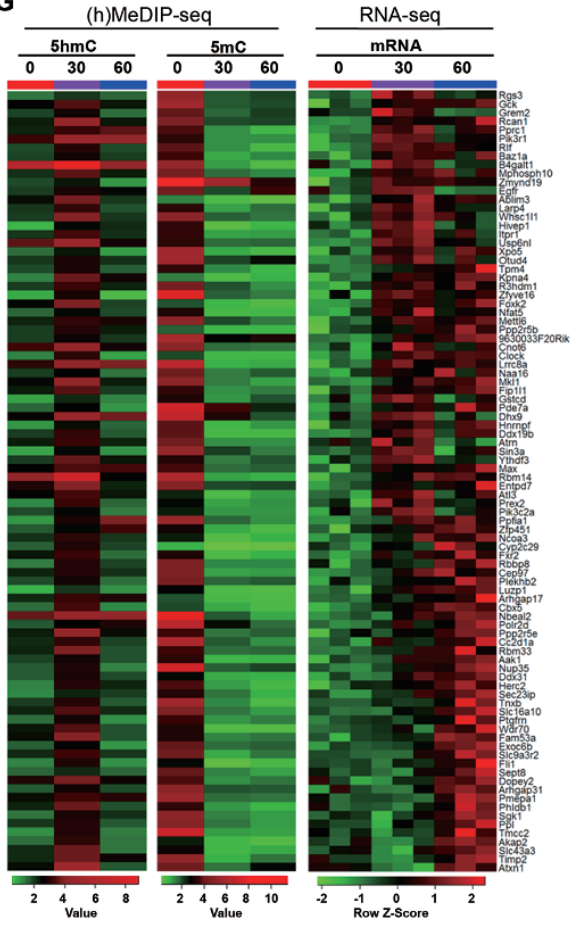

H

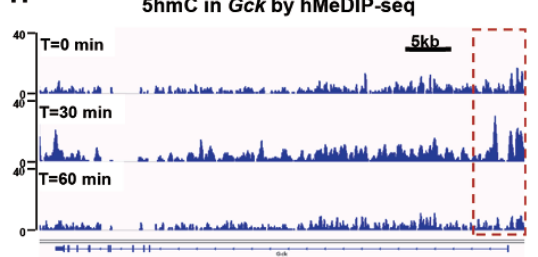

Figure 1 TET-catalyzed $5 \mathrm{hmC}$ production is dynamically regulated by metabolites. (A) The strategy for glucose injection in mice. (B) Relative $\alpha-K G$ levels in mouse livers at the indicated time points after glucose injection, as determined by GC-MS analysis. (C) Relative genome-wide levels of $5 \mathrm{hmC}$ in mouse livers at the indicated time points after glucose injection, as determined by LC-MS/MS analysis. (D) The strategy for glutamine or glutamate injection in mice. (E) Relative $\alpha$-KG levels in mouse livers at the indicated time points after glutamine or glutamate injection, as determined by GC-MS analysis. (F) Relative genome-wide levels of $5 \mathrm{hmC}$ in mouse livers at the indicated time points after glutamine or glutamate injection, as determined by LC-MS/MS analysis. (G) Heatmaps of hMeDIP-seq, MeDIP-deq, and RNA-seq analyses in mouse livers at the indicated time points after glucose injection. Genes were listed, which displayed $\geq 1$. 3 -fold increase in $5 \mathrm{hmC}$ and $\geq 2$-fold decrease in $5 \mathrm{mC}$ at $t=30$ min post glucose injection, and exhibited significant up-regulation in mRNA expression $(P<0.05$ and fold change $>1.2$ at either $t=30$ or $t=60$ min post glucose injection). A red to green color scale indicates values from high to low, and row-scaling is used to facilitate visualization. (H) hMeDIP-seq peaks in the Gck gene visualized in Integrative Genomics Viewer (IGV). One region labeled by a red-dotted box was shown as an enlarged drawing in Supplementary information, Figure S1H. (I) Relative 5hmC levels in one selected region of the Gck gene (chr11:5948151bp$5948348 \mathrm{bp}$ ) in mouse livers at the indicated time points after glucose injection, as determined by hMeDIP-qPCR analysis. (J) mRNA expression of the Gck gene in mouse livers at the indicated time points after glucose injection, as determined by qRT-PCR analysis. Data shown represented the mean \pm SD. (B, C) $n=2-5$ per group; (D-G) $n=3$ per group. ${ }^{*} P<0.05,{ }^{* *} P<0.01,{ }^{* * *} P<0.001$ for comparison with $t=0 \mathrm{~min}$; $\#<0.05, \# \#<0.01$, \#\#\#<0.001 for the indicated comparison. n.s. = not significant.

dicating that glucose shock did not affect the subcellular localization of Tet3 (Supplementary information, Figure $\mathrm{S} 1 \mathrm{~F})$. Therefore, the observed dynamic changes in global $5 \mathrm{hmC}$ levels in mouse tissues after glucose injection are apparently not caused by changes in Tet expression, protein stability, and subcellular localization. Collectively, our results support a notion that in vivo $\alpha-\mathrm{KG}$ is a rate- limiting factor and that an increase in the intracellular concentration of $\alpha-K G$ resulting from glucose injection can rapidly, within $30 \mathrm{~min}$, raise the levels of TET reaction product, $5 \mathrm{hmC}$.

In addition to glycolysis, glutaminolysis can also contribute to $\alpha-K G$ production via converting glutamine to glutamate and then to $\alpha-K G$. To further investigate 
whether a change in $\alpha-K G$ levels may influence TETmediated $5 \mathrm{mC}$ oxidation in vivo, mice were administered with either glutamine or glutamate via an intraperitoneal injection protocol (Figure 1D). GC-MS analysis demonstrated a rapid increase in $\alpha-K G$ levels in mouse livers, which reached peak values at $t=15 \mathrm{~min}$ after glutamine or glutamate injection (Figure 1E). Consistent with dotblot results (Supplementary information, Figure S1G), LC-MS/MS analysis demonstrated that global $5 \mathrm{hmC}$ level was rapidly increased by 1.7 -fold $(P<0.05)$ and 1.6-fold $(P<0.01)$ in mouse livers within 15 min of glutamine and glutamate injection, respectively (Figure $1 \mathrm{~F}$ ). Moreover, we found that a global decrease of $5 \mathrm{mC}$ level paralleled with the increase in $5 \mathrm{hmC}$ level after glutamine or glutamate injection (Supplementary information, Figure S1G). Together, these data further support the idea that $\alpha-\mathrm{KG}$ is a critical determinant of the dynamic nature of $5 \mathrm{hmC}$ in vivo.

Emerging evidence indicates that $5 \mathrm{hmC}$ may directly contribute to gene regulation in addition to being an intermediate in the $5 \mathrm{mC}$ demethylation process. We thus carried out combined RNA-seq and hydroxymethylated DNA immunoprecipitation (hMeDIP)-seq analyses to identify genes whose transcriptional activation in mouse livers after glucose injection was associated with increased $5 \mathrm{hmC}$ and decreased $5 \mathrm{mC}$ (Figure $1 \mathrm{G}$ and supplementary information, Table S1A and S1B). Supporting a functional role of $5 \mathrm{hmC}$ in gene activation, we observed that the Gck gene, which encodes glucokinase (Hexokinase 4), was activated rapidly at $t=30 \mathrm{~min}$ after glucose injection, and this transcriptional activation was associated with remarkably increased $5 \mathrm{hmC}$ and decreased $5 \mathrm{mC}$ levels in the Gck gene (Figure $1 \mathrm{G}-1 \mathrm{H}$ and Supplementary information, Figure S1H). Glucokinase catalyzes the phosphorylation of glucose to glucose6-phosphate (G6P) and plays an important role in the regulation of carbohydrate metabolism by acting as a glucose sensor. Interestingly, $5 \mathrm{hmC}$ at the same regions of Gck was rapidly decreased to an almost basal level at $t=60 \mathrm{~min}$ after glucose injection, while $5 \mathrm{mC}$ was less changed during glucose withdrawal (Figure 1G). In addition to $G c k$, other 89 genes were also identified whose transcriptional activation after glucose shock was found to be related with increased $5 \mathrm{hmC}$ and decreased $5 \mathrm{mC}$ levels (Figure 1G). Moreover, we have identified 732 genes whose mRNA expression was down- or upregulated without significant changes in $5 \mathrm{hmC}$ levels at $t$ $=30$ and $60 \mathrm{~min}$ after glucose injection (Supplementary information, Table S1C and S1D).

To verify these sequencing data, we then performed hMeDIP-qPCR and MeDIP-qPCR analyses to track $5 \mathrm{hmC}$ and $5 \mathrm{mC}$ changes in the selected regions of $\mathrm{Gck}$, respectively. Consistent with the hMeDIP-seq results, hMeDIP-qPCR analysis demonstrated that $5 \mathrm{hmC}$ was rapidly and significantly $(P<0.001)$ increased in one of the CpG-rich regions of Gck in mouse livers at $t=30$ min after glucose injection (Figure 1I). Such a rapid gain of $5 \mathrm{hmC}$ was in parallel with a rapid decrease in $5 \mathrm{mC}$ at the same locus of Gck (Supplementary information, Figure $\mathrm{S} 1 \mathrm{H}$ ). Furthermore, $5 \mathrm{hmC}$ at the same region of Gck was maintained in mouse livers at $t=30+30 \mathrm{~min}$ after the first glucose injection if they received a second injection (Figure 1I). In contrast, $5 \mathrm{mC}$ levels remained at a low level at $t=60 \mathrm{~min}$ after single or repeated glucose injection (Supplementary information, Figure $\mathrm{S} 1 \mathrm{H}$ ). There was no change in $5 \mathrm{hmC}$ and $5 \mathrm{mC}$ levels in a control upstream region of Gck with comparable GC content in mouse livers after glucose injection (Supplementary information, Figure S1I). Finally, qRT-PCR analysis confirmed that the Gck gene became activated in mouse livers as rapidly as $30 \mathrm{~min}$ after glucose injection, and its expression levels were further upregulated by repeated glucose injection (Figure 1J). The expression of other glycolytic or gluconeogenic genes was, however, not significantly changed after glucose shock (Supplementary information, Figure S1J). Taken together, these results suggest that $5 \mathrm{hmC}$ can undergo dynamic changes, which is associated with the regulation of specific gene expression in response to altered cellular metabolic conditions.

How stable $5 \mathrm{hmC}$ modifications are in the genome is currently not known, but could have broad implications to the understanding of epigenetic control and gene regulation. One view holds that while the production of $5 \mathrm{hmC}$ is enzymatically catalyzed, its decline or loss in preimplantation embryos is passively accomplished through a replication-coupled dilution mechanism [12]. Our results presented here reveal two novel features of $5 \mathrm{hmC}$ regulation. First, TET-catalyzed genome-wide $5 \mathrm{hmC}$ production can be dynamically regulated, and $5 \mathrm{hmC}$ decline can occur independently of DNA replication. Second, we provide evidence that $\alpha-K G$, the co-substrate of TET enzymes and a key TCA metabolite, appears to be a critical factor in the regulation of $5 \mathrm{hmC}$ dynamics in vivo. Following the injection of glucose, glutamine or glutamate, the concentration of $\alpha-\mathrm{KG}$, which is dynamically and tightly regulated in response to cellular metabolic conditions, exhibits a pattern of increase/decrease that is closely correlated with that of $5 \mathrm{hmC}$. The physiological significance of $5 \mathrm{hmC}$ dynamics is supported by the finding that $5 \mathrm{hmC}$ and $5 \mathrm{mC}$ levels in the promoter regions of Gck gene, a glycogen synthesis pathway gene, were rapidly increased and decreased, respectively, following high glucose shock. An important implication of this study is that besides developmental controls where 
long-term stable gene silencing or derepression is more desirably achieved by the regulation of $5 \mathrm{mC}$ and $5 \mathrm{hmC}$, additional cellular cues, such as metabolic conditions, could also influence the TET enzymes to regulate the status of $5 \mathrm{mC} / 5 \mathrm{hmC}$ at specific loci and thus the expression of responsible genes. Moreover, we cannot exclude the possibility that glucose may affect DNA epigenetic modification by other mechanisms. A recent study reported that the nuclear localization and O-GlcNAcylation of TET3 was regulated by glucose metabolism [13], which was, however, not observed in our current study. It is also possible that glucose may affect the DNA repair pathway and thus demethylation. We show here that the increase of $5 \mathrm{hmC}$ can be maintained by repeated glucose injection, indicating that a hyper-5hmC level may be sustained by high glucose. How $5 \mathrm{hmC}$ is affected under hyperglycemia conditions and plays a role in gene regulation is another interesting question that warrants future investigations.

\section{Acknowledgments}

We thank the members of the Fudan MCB laboratory (Lisha Zhou, Shenghong Ma, Mengtao Xiao, Shiwen Wang) and China Novartis Institutes for BioMedical Research (Tengfei Zhang, Qi Fei, Yuting Liu) for discussion and support throughout this study. We thank the staff members of Biomedical Core Facility, Fudan University for their help on mass spectrometry analyses. This work was supported by the National Basic Research Program of China (973 Program; 2012CB910303, 2012CB910101, 2011CB910600 and 2009CB918401), the Innovation Program of Shanghai Municipal Education Commission (12ZZ008), and the Scholarship Award for Excellent Doctoral Student granted by the Ministry of Education (JFH1340003). This work was also supported by NIH grants (CA163834 to YX and R01CA108941 to KLG), and Samuel Waxman Foundation (to YX).

Hui Yang ${ }^{1}$, Huaipeng Lin ${ }^{1}$, Haiyan $\mathrm{Xu}^{2}$, Lei Zhang ${ }^{3}$, Lu Cheng ${ }^{4}$, Bo Wen ${ }^{4}$, Jianyong Shou ${ }^{2}$, Kunliang Guan ${ }^{1,5}$, Yue Xiong ${ }^{1,6}$, Dan $\mathrm{Ye}^{1}$

${ }^{I}$ Molecular and Cell Biology Lab of Key Laboratory of Molecular Medicine of Ministry of Education and Institutes of Biomedical Sciences, Shanghai Medical College, College of Life Science, Fudan University, Shanghai 200032, China $;^{2}$ China Novartis Institutes for BioMedical Research, Shanghai, China; ${ }^{3}$ Department of Chemistry, ${ }^{4}$ Department of Biochemistry and Molecular Biology, Institute of Stem Cell Research and Regenerative Medicine, Fudan University, Shanghai 200032, China; ${ }^{5}$ Department of Pharmacology and Moores Cancer Center, University of California San Diego, La Jolla, California 92093, USA; ${ }^{6}$ Lineberger Comprehensive Cancer Center, Department of Biochemistry and Biophysics, University of North Carolina at Chapel Hill, North Carolina 27599, USA

Correspondence: Dan Ye

E-mail: yedan@fudan.edu.cn

\section{References}

1 Cedar H, Bergman Y. Nat Rev Genet 2009; 10:295-304.

2 Jones PA, Baylin SB. Cell 2007; 128:683-692.

3 He YF, Li BZ, Li Z, et al. Science 2011; 333:1303-1307.

4 Ito S, D'Alessio AC, Taranova OV, et al. Nature 2010; 466:1129-1133.

5 Ito S, Shen L, Dai Q, et al. Science 2011; 333:1300-1303.

6 Kriaucionis S, Heintz N. Science 2009; 324:929-930.

7 Takahashi K, Yamanaka S. Cell 2006; 126:663-676.

8 Loenarz C, Schofield CJ. Trends Biochem Sci 2011; 36:7-18.

9 Rose NR, McDonough MA, King ON, et al. Chem Soc Rev 2011; 40:4364-4397.

10 Killian JK, Kim SY, Miettinen M, et al. Cancer Discov 2013; 3:648657.

11 Figueroa ME, Abdel-Wahab O, Lu C, et al. Cancer Cell 2010; 18:553567.

12 Inoue A, Zhang Y. Science 2011; 334:194-194.

13 Zhang Q, Liu X, Gao W, et al. J Biol Chem 2014; 289:5986-5996.

(Supplementary information is linked to the online version of the paper on the Cell Research website.) 